\title{
Puerperal psychosis: identifying and caring for women at risk
}

\author{
Ian Jones \& Sue Smith
}

\begin{abstract}
SUMMARY
Puerperal (postpartum) psychosis - the acute onset of a manic or psychotic episode shortly after childbirth - most commonly occurs in women with a bipolar disorder diathesis who have a vulnerability to a specific childbirth-related trigger. Women with bipolar disorder are at particularly high risk of puerperal psychosis, with a severe affective episode following between 25 and $50 \%$ of deliveries. Suicide is a leading cause of maternal death in the UK and it is clear that we must do more to identify and better manage women at high risk of illness related to childbirth. The clinical picture of puerperal psychosis can vary dramatically from hour to hour and can escalate quickly to a true psychiatric emergency. It is vital that clinical services identify women who are unwell and can respond quickly to the severity of illness, delivering treatment in the most appropriate setting for the mother and her baby.
\end{abstract}

\section{DECLARATION OF INTEREST}

I.J. has received honoraria or consultancy fees from Lilly, GlaxoSmithKline, Lundbeck, Janssen and AstraZeneca and research funding from GlaxoSmithKline.

The concept of puerperal or postpartum psychosis has a long history, but has fallen somewhat into disrepute in the age of ICD and DSM classifications that do not recognise this disorder as a nosological entity. The importance of this condition is underlined, however, by a number of tragic cases in which women experiencing puerperal psychosis have taken their own lives or harmed their baby (Jones 2005). The past three confidential enquiries into maternal deaths in the UK have found suicide to be a leading cause of maternal death (Lewis 2001, 2004, 2007) and it is clear that a high proportion of maternal suicides occur in women with an acute onset of psychosis in the early postpartum period (Oates 2003a). Therefore, the stakes are high and emphasise the importance of the early recognition and prompt treatment of women who become ill.

In this review we will pose, and attempt to answer, a number of questions in relation to puerperal psychosis. We will consider first what constitutes an episode of this disorder, then what is known about its aetiology and finally focus our attention on how to identify and care for women at risk.

\section{What affective disorders occur in relation to childbirth?}

More than $50 \%$ of women experience transient episodes of emotional lability following childbirth (Heron 2005) but these symptoms last only a few days, do not require intervention and should not be labelled as a disorder. Episodes of more clinically significant depression, however, commonly occur both in pregnancy and following delivery and may last many months, causing significant disruption to the woman and her family. Many studies have been conducted in a variety of settings and employing differing definitions of postnatal depression and show $10-15 \%$ of mothers to be depressed at some time during the 6 months following childbirth, although a much lower figure (perhaps 2-4\%) receive treatment (Brockington 1996: pp. 200-284).

The term 'puerperal psychosis' usually refers to a severe mental illness with a dramatic onset shortly after childbirth - the majority in the first few postpartum days (Heron 2007). Although there may be little difficulty identifying what constitutes the 'core' of this phenomenon, problems occur when considering its boundaries and the concept is difficult to tie down, with a great variety of definitions employed both clinically and in research.

\section{What episodes are included in the concept of puerperal psychosis?}

A wide range of diagnoses can present with psychotic features in the puerperium and include acute confusional states due to a variety of causes (e.g. eclampsia or infection), alcohol withdrawal, chronic psychosis (e.g. schizophrenia), psychotic depression, mania, and schizophreniform and schizoaffective episodes. The term puerperal psychosis is usually reserved for the acute onset of an episode of severe affective psychosis, including manic, depressive and schizoaffective forms in a woman who has previously been well, even though she may have a previous history.
ARTICLE

an Jones is a senior lecturer in perinatal psychiatry and honorary consultant perinatal psychiatrist in the Department of Psychological Medicine at Cardiff University. Sue Smith is a consultant psychiatrist in the Cardiff and Vale National Health Service Trust and consultant perinatal psychiatrist for the Mother and Baby Unit in Cardiff. Correspondence Dr lan Jones, Department of Psychological Medicine, School of Medicine, Cardiff University, Henry Wellcome Building for Biomedical Research, Academic Avenue, Heath Park, Cardiff CF14 4XN, UK. Email: jonesir1@cf.ac.uk 
Perhaps even greater problems arise when considering the temporal relationship of episodes to childbirth. Time frames have varied widely in research studies, from a tight definition with onset within 2 weeks of delivery to studies that have included all women found to experience psychosis within the first postpartum year. It is usual, however, to limit the concept of puerperal psychosis to episodes with onset shortly following delivery, within weeks rather than months.

\section{How is puerperal psychosis treated in the current classification systems?}

There is little consensus regarding the nosological status of episodes of psychotic illness in the puerperium, some of the typical clinical symptoms of which are described in Box 1. Opinion has varied from those who have argued that puerperal psychosis is a condition in its own right to those who have regarded childbirth as a non-specific stressor like any other life event, acting to trigger a wide variety of psychotic illness (for a review of this debate see Brockington 1996: pp. 200-284).

The non-specific nature of puerperal episodes has become the predominant view over the past 20 years, a view that is reflected in, and moulded by, the way puerperal episodes are dealt with in the ICD and DSM classification systems.

Although postpartum psychosis was included in ICD-8 (World Health Organization 1974), its use was qualified by the instruction only to use this diagnosis when the use of another category was not possible. The category disappeared in ICD-9 and 10 (World Health Organization 1978, 1992), with only a ragbag 'mental and behavioural disorders associated with the puerperium, not elsewhere classified' available for episodes with onset within 6 weeks of delivery and only if they do not meet the criteria for disorders classified elsewhere.

DSM-II (American Psychiatric Association 1968) included a category of postpartum psychosis but again carried the instruction to use only if 'all other possible diagnoses have been excluded'. In DSM-IV (American Psychiatric Association 1994), although the category has disappeared, a

\footnotetext{
B0X 1 Clinical symptoms typical of puerperal psychosis

- A wide variety of psychotic phenomena such as delusions and hallucinations, the content of which is often related to the new child

- Affective (mood) symptoms, both elation and depression
}

'postpartum onset specifier' is included in the mood disorders chapter that could be applied for onsets within 4 weeks of delivery. Modern classification systems, therefore, do not recognise puerperal psychosis as a separate nosological entity, but in DSM at least, these episodes are treated as mood disorders with a postpartum trigger. It is clear, however, that the term postpartum or puerperal psychosis has remained in clinical use and that the nosological confusion of current classification systems has hindered research into this important disorder.

\section{Is childbirth a period of increased risk and is puerperal psychosis related to any particular diagnosis?}

Despite difficulties in the concept and classification of postpartum psychosis, it is clear that childbirth is a potent trigger of severe psychiatric episodes. Doubts have been raised regarding the increased risk of episodes of non-psychotic depression following childbirth. In contrast, a robust research base demonstrates that the puerperium is a time of considerably increased risk of severe manic or psychotic mood disorder. The UK studies of Kendell and colleagues in Camberwell (south London) and Edinburgh found a significant increase in rates of psychiatric admission following childbirth - women were at a 22 -fold increased risk of having an episode of affective psychosis in the 4 weeks following delivery; the risk was even higher (relative risk $\mathrm{RR}=35$ ) when only first deliveries were considered (Kendell 1987).

However, the study of Terp \& Mortensen (1998) linking Danish birth and psychiatric admission registers for more than a million births found seemingly different results. It reported only a small increase in the risk of admission with an episode of functional psychosis in the three months following delivery ( $\mathrm{RR}=1.09 ; 95 \% \mathrm{CI} 1.03-1.16)$, although this figure was much higher for first admissions $(\mathrm{RR}=3.21 ; 95 \% \mathrm{CI}$ 2.96-3.49). When individual disorders were considered, however, the findings were far closer to those of the UK studies (Kendell 1987). The risk of psychosis following childbirth was not distributed evenly across the functional psychoses - the chances of being admitted with an episode of schizophrenia were actually lower than at other times, whereas women with bipolar disorder were at particularly high risk. Indeed, the highest relative risk (6.82) was obtained for first-episode bipolar psychosis 2-28 days after delivery and, although not reported in the original article, an even higher figure (approaching 20) was obtained if only the first 2 weeks of the puerperium were considered. Moreover, the 
analysis of diagnostic subgroups in the Danish study ignored a large number of women who had a diagnosis of ICD-8 'puerperal psychosis', as no equivalent non-puerperal diagnosis was available. Given that it is likely that the majority of women diagnosed with puerperal psychosis would have a bipolar-spectrum disorder, the relative risk for bipolar disorder is likely to be an underestimate of the increased risk. The true magnitude of risk to women with bipolar disorder is underlined by a further analysis of the Danish data, which reports the relative risk for an admission with bipolar disorder in the month following first pregnancies to be 23 - more than four times higher than the relative risk for admission with schizophrenia (Munk-Olsen 2006).

\section{A link with bipolar disorder?}

Despite the wide variety of clinical features seen in episodes of puerperal psychosis, there is indeed strong evidence from clinical, outcome and genetic studies for a close relationship with bipolar disorder. There are marked variations in inclusion criteria and definitions of the puerperal period, but studies consistently demonstrate that the majority of puerperal psychotic episodes are affective, with mania particularly common in the 2 weeks following childbirth (Brockington 1996: pp. 200-284). Brockington and colleagues (1981) examined the symptoms experienced in 58 puerperal episodes compared with 52 episodes of non-puerperal psychotic illness occurring in women of childbearing age. They found that systematisation of delusions, persecutory ideas, auditory hallucinations, odd affect and social withdrawal were less common in the puerperal patients, whereas manic symptoms - elation, rambling speech, flight of ideas, lability of mood, distractibility, euphoria and excessive activity were all more frequent and severe.

Further evidence for a close link to bipolar disorder comes from studies examining the natural history of puerperal psychotic episodes. The excellent prognosis associated with this condition has been noted for hundreds of years but women remain at high risk of developing further puerperal and non-puerperal affective episodes (Robertson 2005). A number of studies have focused on women with bipolar disorder and have found this group to be at particular risk in the puerperium, with episodes following $25-50 \%$ of deliveries (Jones 2001a).

In summary, although the postpartum period may not be a time of high risk for all functional psychoses, it is clearly a time of substantial risk for episodes of bipolar disorder.

\section{How common is puerperal psychosis?}

Although this may appear a relatively simple question, it is, in fact, difficult to answer because of the various definitions of puerperal psychosis that can be used. The studies of Kendell and colleagues (1987) mentioned above identified newonset psychotic episodes in 1 in 250 deliveries in south London and found an admission rate of 1 in 500 deliveries in Edinburgh. There was a wide range of diagnoses in women admitted, however, and limiting to clearly psychotic diagnoses gives a rate of about 1 in 1000 deliveries; however, it is probable that a proportion of the episodes of depression were psychotic and therefore a higher rate is likely. The Danish register study involving more than a million deliveries found a similar admission rate of 0.99 for every 1000 deliveries in the first 3 postpartum months (Terp 1998).

The true incidence rates may be higher than in those studies focusing on admission rates, as a number of women with significant puerperal episodes are likely to be treated at home particularly if facilities for admission with the baby are not available.

\section{What is the prognosis for puerperal psychotic episodes?}

The numerous follow-up studies of severe puerperal episodes suggest that, although the prognosis for recovery from the initial episode is excellent, women remain at risk of subsequent puerperal and non-puerperal episodes. Recurrence rates following subsequent pregnancies are 50\% or more, and about $50 \%$ of women have further non-puerperal episodes (Robertson 2005).

\section{How should we consider the status of puerperal psychosis?}

Although the status of puerperal psychosis and its clinical boundaries remain subject to debate, the weight of evidence supports a close link to bipolar illness and it is clear that women with a bipolar diathesis are at particularly high risk of episodes in the puerperium. Perhaps it is most helpful therefore, in clinical practice and in research, to consider most episodes of puerperal psychosis as affecting women with a bipolar disorder diathesis acted on by a specific puerperal trigger.

\section{What makes some women vulnerable to bipolar episodes following pregnancy?}

One avenue that may prove productive in research is the search for genetic factors that increase vulnerability to the puerperal triggering of episodes of bipolar disorder. Evidence from family 
studies suggests that vulnerability to affective disorders is increased in the relatives of women who experience puerperal psychosis (Jones 2001b). Moreover, studies have suggested that puerperal psychosis is a marker for a more familial form of bipolar disorder (Jones 2002) and that a specific vulnerability to the puerperal triggering of bipolar illness is familial (Jones 2001a). Further support for the involvement of genetic factors comes from the report of familial clustering of puerperal psychosis associated with consanguinity, which raises the possibility that a recessive gene contributes to susceptibility (Craddock 1994). Molecular genetic studies of puerperal psychosis are ongoing, with interesting findings at the serotonin transporter gene (Coyle 2000) and linkage evidence pointing to the long arm of chromosome 16 (Jones 2007).

The relationship between genetic factors influencing puerperal triggering and those for the bipolar diathesis remain unclear. It is possible that one or more susceptibility genes for bipolar illness also lead to a vulnerability to puerperal triggering. Alternatively, the genetic factors influencing puerperal vulnerability may be completely distinct from those that determine the bipolar diathesis and act as course modifiers. It is likely that only when the genetic factors are found will the relationship be resolved.

\section{What is the nature of the trigger?}

\section{Parity}

Clues to the aetiology of puerperal triggering have been sought in epidemiological studies and a number of potential risk factors have emerged. After a personal and family history of psychiatric illness, perhaps the best established risk factor for episodes of puerperal psychosis is parity episodes being more common following first babies (Robertson Blackmore 2006). The reason for the excessive risk in primiparous women is not clear. An important bias is that women with a severe postpartum episode may be less likely to go on to have further children but further analyses suggest that this is unlikely to be the sole, or even the main, explanation. Another possible explanation is that first pregnancies are a greater psychosocial stressor than subsequent deliveries but, in contrast to postnatal depression, psychosocial factors have not been shown to play a major role in vulnerability to psychosis in the puerperium. Four studies have examined the characteristics of high-risk women who experience a puerperal psychotic episode and are consistent in finding no association with stressful life events (McNeil 1988; Brockington 1990, Dowlatshahi 1990; Marks 1991).
The possibility remains, therefore, that the effect of primiparity is, at least in part, due to biological differences between first and subsequent pregnancies. Hormonal, immunological and other biological differences between first and subsequent pregnancies are therefore interesting targets for further investigation into the aetiology of puerperal psychosis (Robertson Blackmore 2006).

\section{Obstetric factors}

A further area that has received attention is the possibility that certain obstetric factors are associated with episodes of puerperal psychosis, with both obstetric complication and Caesarean section rates found to be higher in some studies. In 2006, we compared affected and unaffected deliveries in more than 50 women with puerperal psychosis and found that experiencing a complication during delivery more than doubled the risk of puerperal psychosis (Robertson Blackmore 2006). Other factors that have been examined such as the gender of the child and gestation of pregnancy have not been consistently supported as risk factors.

\section{Hormonal factors}

The lack of evidence implicating psychosocial factors and consideration of the abrupt onset during a time of major physiological change suggests that biological, possibly hormonal, factors are of fundamental importance. The role of several hormones (e.g. progesterone, prolactin, follicular stimulating hormone (FSH) and luteinising hormone (LH)) has been considered but oestrogen has perhaps received the greatest attention.

A number of studies have examined a range of hormonal measures in women with postpartum affective episodes and in controls (Hendrick 1998; Bloch 2003), but no consistent well-replicated hormonal differences have been demonstrated. It is likely, therefore, that women with puerperal psychotic episodes do not show gross abnormalities in endocrine physiology but rather that a vulnerability to puerperal triggering represents an abnormal response to the normal hormonal fluctuations of pregnancy and childbirth.

The evidence pointing to reproductive hormones in the aetiology of puerperal triggering is predominantly circumstantial. One study, however, provides more direct evidence for the involvement of oestrogen and progesterone in the puerperal triggering of affective symptoms. Bloch and colleagues (2000) employed a paradigm in which they simulated the supraphysiological gonadal steroid levels of pregnancy and withdrawal to a hypogonadal state in eight women with and 
eight women without a history of postpartum depression. They found that five of the eight women with a history of postpartum depression and none of the women in the comparison group developed significant mood symptoms during the withdrawal period.

\section{Withdrawal of mood stabilisers}

Although hormonal theories of the aetiology of puerperal triggering have received the greatest attention, other explanations are possible. Viguera and colleagues (2000) examined the hypothesis that the excess of episodes of bipolar illness seen after delivery could be accounted for by the fact that women with bipolar disorder often come off medication, such as lithium, prior to conception or in early pregnancy because of concerns over toxicity to the fetus. They employed survival analysis to examine the course of 42 women with bipolar disorder who stopped taking lithium because of pregnancy compared with 59 agematched non-pregnant women who discontinued lithium. Rates of recurrence were very similar for both groups up to 40 weeks but following delivery, in weeks 41 to 64 , a large and highly significant difference in recurrence rates was observed ( $70 \%$ v. $24 \%$ recurrence). The increased risk of recurrence following childbirth for women with bipolar disorder is not, it appears, merely a result of stopping mood-stabilising medication.

\section{Sleep deprivation}

A plausible hypothesis that has received surprisingly little attention in the literature is that the sleep deprivation of delivery and the immediate postpartum period is responsible for puerperal triggering of illness (Sharma 2004). It has been suggested that sleep loss can effectively trigger the onset of mania in people with bipolar disorder and it does not take an extensive research effort to know that sleep loss is common for new mothers. Intuitively, sleep disturbance is an excellent candidate for involvement in the puerperal trigger but there is little evidence to support this hypothesis.

\section{Why is puerperal psychosis important?}

Great strides have been taken in reducing childbirth-related deaths in areas such as haemorrhage and infection. This has left suicide as one of the leading causes of maternal mortality in the UK (Lewis 2001, 2004, 2007). The majority of the mothers who die by suicide experience an abrupt onset of a severe psychotic illness within days of childbirth - puerperal psychosis under our definition above. In addition, for a significant proportion of women the episode of puerperal psychosis is predictable from their previous history. The 2001 Confidential Enquiry into Maternal Deaths in the UK, for example, recorded that $46 \%$ of the women who took their own life had previous contact with psychiatric services and half of these had previously been admitted to hospital with a severe episode of illness following childbirth (Oates 2003b).

\section{How can women vulnerable to puerperal psychosis be identified?}

As we have seen, a compelling literature suggests that women with bipolar disorder are at particularly high risk of puerperal psychosis. This high rate of illness represents a many hundred-fold increase from the base rate of about 1 in 1000 deliveries (Fig. 1). In addition to a history of bipolar disorder, other important risk factors include having experienced a previous episode of puerperal psychosis, having a first-degree relative who has experienced one and having a firstdegree relative with bipolar disorder (Jones 2005). However, because of the relapsing and remitting nature of bipolar disorder, women at high risk are often currently well and not in contact with mental health services and will fail to recognise the serious risks of the situation. Various reports and enquiries since 2005, including guidelines published by the National Institute for Health and Clinical Excellence(NICE) (National Collaborating Centre for Mental Health 2006, 2007; National Collaborating Centre for Women's and Children's Health 2008), have therefore recommended that all antenatal women be screened for known important risk factors and protocols be put in place to ensure that women at potential risk receive a formal risk assessment and management plan (Jones 2005). How screening and risk management are delivered will differ according to local circumstances but

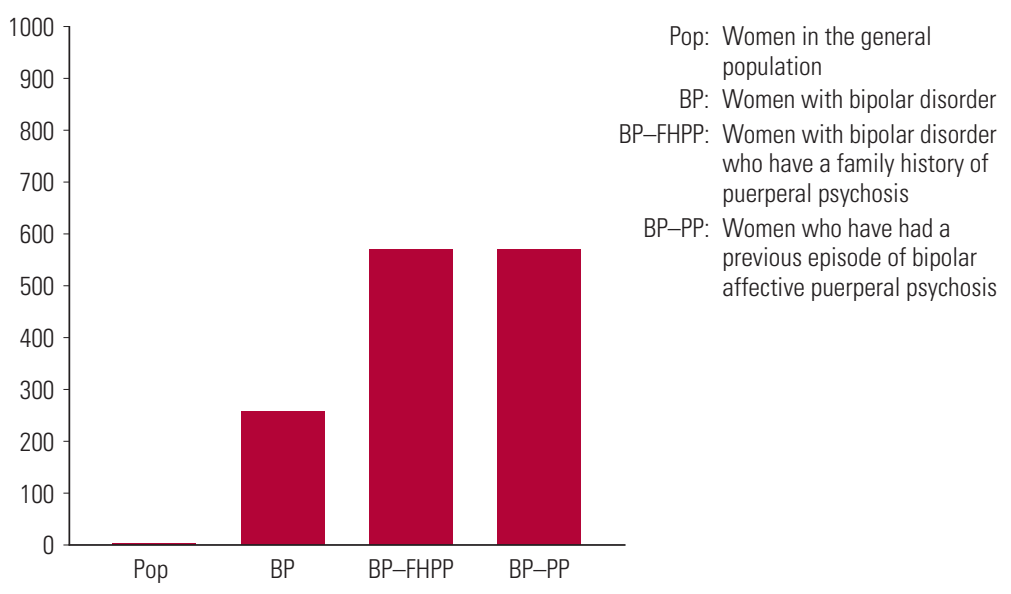

FIG 1 Rates of puerperal psychosis for every 1000 deliveries (Jones 2001a; Robertson 2005). 
${ }^{\dagger}$ For further discussion in Advances see Kohen D (2004) Psychotropic medication in pregnancy. 10: 59-66 Ed.

they are arguably best achieved by a specialist obstetric liaison team as part of a comprehensive perinatal psychiatric service.

\section{How should women at risk of puerperal psychosis be cared for?}

Women at high risk of puerperal psychosis need very careful care before conception, throughout pregnancy and during the postpartum period (Key points 1). The high risk of illness in the 2 weeks following delivery in a woman with a history of bipolar disorder must be recognised both by healthcare professionals and by the woman herself. The possibility of future pregnancy should be considered in all women with bipolar disorder who are of childbearing age. The risks of illness following childbirth should be discussed with women and the importance of seeking help if contemplating pregnancy (or if unexpectedly becoming pregnant) emphasised. Decisions about continuing or stopping medications before, or during, pregnancy are difficult and should be the result of a detailed and individualised costbenefit analysis. Although there are significant concerns about the reproductive safety of many of the medications used to treat individuals with bipolar disorder, the risks of stopping medication must also be considered. ${ }^{\dagger}$ In a study in 2007, for example, women with bipolar disorder who stopped medication during pregnancy were more than twice as likely to experience a recurrence of the disorder than those who remained on medication (Viguera 2007a).

\section{Medications for bipolar disorder}

With regard to specific medications, the weight of evidence suggests that the use of sodium valproate in pregnancy involves a substantial risk

\section{KEY POINTS 1 Caring for women at high risk of puerperal psychosis}

- Care for all women in their reproductive years with pregnancy in mind

- Recognise the high risk of postpartum episodes in women with bipolar disorder

- Regularly discuss contraception and risks of illness following childbirth with women with bipolar disorder

- Decisions about individual medications in pregnancy are difficult and should be made in light of a full risk-benefit analysis

- Although there are reproductive safety concerns over a range of psychotropic medications (e.g. lithium and carbamazepine), there are particular concerns over sodium valproate

- Women at high risk must be identified in pregnancy and the mainstay of management is keeping them under close review through the perinatal period and responding quickly to evidence of illness

- The severity and unpredictability of psychotic illness in the puerperium mean that admission is usually necessary - preferably with the baby in a mother and baby unit of major congenital malformations and shows a clear association with later cognitive impairment to children exposed in utero. This has led the NICE (National Collaborating Centre for Mental Health 2006, 2007) to single out this medication, not only advising strongly against its use by pregnant women but also stating that it should not be routinely used by women of childbearing potential. In those rare situations where there are considered to be no alternative options, women should be counselled strongly about the need to avoid accidental pregnancy.

It is clear that there are also reproductive safety issues for other drugs used in the long-term management of bipolar disorder. Lithium and carbamazepine are likely to increase malformation rates although, particularly for lithium, we still have very few data on the magnitude of the increased risk. Moreover, the management of lithium in pregnancy is complex and it should therefore be used only if all other options have been rejected.

For atypical antipsychotics, although there is no evidence of reproductive safety issues at present, again we have very few data to inform decisions about their use in this context.

No universal recommendations can therefore be made and it must be emphasised that the decision ultimately must rest with the woman and her family. However, stopping medication should always be a carefully considered decision and never a reflex response. Likewise, the decision to start medication for women who develop symptoms in pregnancy or when breastfeeding must be the result of weighing up both the potential risks from taking medication and the risks posed by the illness itself.

\section{General recommendations}

For women at risk, perhaps the most important aspect of care is to maintain close contact and review during the perinatal period. It may also be important to address other avoidable factors that may increase risk - such as decreasing general levels of stress and paying attention to sleep in late pregnancy and the early postpartum weeks. For women with a history of bipolar disorder who have been off medication in pregnancy the introduction of prophylactic medication in the immediate postpartum period should be considered. Some evidence exists for the use of lithium in this context (e.g. Stewart 1991), but the few studies have been open and retrospective and there are practical problems with reaching therapeutic levels quickly to cover the period of risk. These issues have led some perinatal psychiatrists to use typical or atypical antipsychotics as prophylaxis. Despite 
anecdotal reports of success with this strategy, nothing has yet been reported in the literature. Well-conducted trials are needed to assess the use of medication in this context.

\section{What principles should guide the management of puerperal psychosis?}

Ideally, the management of an episode should have started prior to its onset. As discussed above, systems should be in place to identify women at high risk and a plan developed for what to do if symptoms develop despite prophylactic intervention. For $50 \%$ or more of women who develop puerperal psychosis, however, there is no family or personal history that would have put them in a high-risk group. By the time the clinical picture raises the suspicions of family and close friends that something is not right the woman may quickly become extremely ill. Even then the presentation may vary significantly from hour to hour and the immediate clinical impression can often mislead. In most cases the severity and unpredictability of the illness means that even women with the most supportive of families are likely to require hospital admission. The new NICE guidelines for antenatal and postnatal mental health (National Collaborating Centre for Mental Health 2007) state that women who need in-patient care for a mental disorder within a year of childbirth should be admitted to a specialist mother and baby unit unless there are specific reasons for not doing so. However, the provision of services across the UK is patchy and for the majority of women with puerperal psychosis there is no option of admission with their baby.

\section{Are there any specific treatments and how do they affect breastfeeding? ${ }^{\ddagger}$}

The treatment used while the woman is breastfeeding will depend on a number of factors, including the symptoms that she experiences, her level of disturbance and her previous response to medication. The whole range of psychotropic medication may need to be employed, including antidepressants, antipsychotics, mood stabilisers and benzodiazepines. For many women the severity of the illness and the chaotic presentation means breastfeeding becomes impossible anyway. If breastfeeding is being considered, factors in the baby such as prematurity and systemic illness should be considered in addition to the particular properties of the medication itself. Limited data suggest that the use of lithium during breastfeeding is not as problematic as once thought (Viguera 2007b) but it tends to be avoided because of the risk of toxicity in the infant.
Anecdotally, electroconvulsive therapy has often been described as being particularly effective in the treatment of puerperal psychosis and there is limited evidence to support this opinion (Reed 1999). There is certainly an argument that the quicker the mother's symptoms can be brought under control, the less the disruption of the mother-baby bond.

\section{What should women be told about the prognosis and the risk of recurrence?}

The short-term prognosis for puerperal psychosis is generally good. However, women need to be counselled about the risks they run of a further puerperal or non-puerperal episode. This will include discussing the need for longer-term moodstabilising medication and other measures that can reduce the risk of recurrence. Despite the high risk of recurrence following further deliveries, many women make the decision to become pregnant again and it is our strongly held view that women with puerperal psychosis, or indeed bipolar disorder more generally, should not be told that they should not have children. Sadly, it is our experience that many women are still given this advice by healthcare professionals.

\section{References}

American Psychiatric Association (1968) Diagnostic and Statistical Manual of Mental Disorders (2nd edn) (DSM-II). APA.

American Psychiatric Association (1994) Diagnostic and Statistical Manual of Mental Disorders (4th edn) (DSM-IV). APA.

Bloch M, Daly RC, Rubinow DR (2003) Endocrine factors in the etiology of postpartum depression. Comprehensive Psychiatry, 44: 234-46.

Bloch M, Schmidt PJ, Danaceau M, et al (2000) Effects of gonadal steroids in women with a history of postpartum depression. American Journal of Psychiatry, 157: 924-30.

Brockington IF, Cernick KF, Schofield EM, et al (1981) Puerperal psychosis Phenomena and diagnosis. Archives of General Psychiatry, 38: 829-33.

Brockington IF, Martin C, Brown GW, et al (1990) Stress and puerperal psychosis. British Journal of Psychiatry, 157: 331-4.

Brockington IF (1996) Motherhood and Mental Health. Oxford University Press.

Coyle N, Jones I, Roberston E, et al (2000) Variation at the serotonin transporter gene influences susceptibility to bipolar affective puerperal psychosis. Lancet, 356: 1490-1.

Craddock N, Brockington I, Mant R, et al (1994) Bipolar affective psychosis associated with consanguinity. British Journal of Psychiatry, 164: 359-64

Dowlatshahi D, Paykel ES (1990) Life events and social stress in puerperal psychosis. Absence of effect. Psychological Medicine; 20: 655-62.

Hendrick V, Altshuler LL, Suri R (1998) Hormonal changes in the postpartum and implications for postpartum depression. Psychosomatics; 39 93-101

Heron J, Craddock N, Jones I (2005) Postnatal euphoria. Are 'the highs' an indication of bipolarity? Bipolar Disorders; 7: 103-10.

Heron J, Robertson Blackmore E, McGuinness M, et al (2007) No 'latent period' in the onset of bipolar affective puerperal psychosis. Archives of Women's Mental Health; 10: 79-81.

$\begin{array}{lllll}\text { MCO answers } & & \\ 1 & 2 & 3 & 4 & 5 \\ \text { af } & \text { af } & \text { af } & \text { af } & \text { af } \\ \text { bt } & \text { bf } & \text { bf } & \text { bf } & \text { bf } \\ \text { cf } & \text { cf } & \text { cf } & \text { ct } & \text { cf } \\ \text { df } & \text { dt } & \text { df } & \text { df } & \text { dt } \\ \text { ef } & \text { ef } & \text { et } & \text { ef } & \text { ef }\end{array}$

MCO answers

a $a f$

t bf bf bf bf

$d f d t \quad d f d f d t$

ef 
Jones I, Craddock N (2001a) Familiality of the puerperal trigger in bipolar disorder. Results of a family study. American Journal of Psychiatry, 158: 913-7.

Jones I, Lendon C, Coyle N, et al (2001b) Molecular genetic approaches to puerperal psychosis. Progress in Brain Research; 133: 321-32.

Jones I, Craddock N (2002) Do puerperal psychotic episodes identify a more familial subtype of bipolar disorder? Results of a family history study. Psychiatric Genetics; 12: 177-80.

Jones I, Craddock N (2005) Bipolar disorder and childbirth. The importance of recognising risk. British Journal of Psychiatry, 186: 453-4.

Jones I, Hamshere ML, Nangle JM, et al (2007) Bipolar affective puerperal psychosis. Genome-wide significant evidence for linkage to chromosome 16. American Journal of Psychiatry, 164: 1099-104.

Kendell RE, Chalmers JC, Platz C (1987) Epidemiology of puerperal psychoses. British Journal of Psychiatry, 150: 662-73.

Lewis G, Drife J (eds) (2001) The Confidential Enquiry into Maternal and Child Health (CEMACH). Why Mothers Die, 1997-1999. The Fifth Report on Confidential Enquiries into Maternal Deaths in the United Kingdom. RCOG Press.

Lewis G (ed) (2004) The Confidential Enquiry into Maternal and Child Health (CEMACH). Why Mothers Die, 2000-2002. The Sixth Report on Confidential Enquiries into Maternal Deaths in the United Kingdom. RCOG Press.

Lewis, G (ed) (2007) The Confidential Enquiry into Maternal and Child Health (CEMACH). Saving Mothers' Lives: Reviewing Maternal Deaths to Make Motherhood Safer-2003-2005. The Seventh Report on Confidential Enquiries into Maternal Deaths in the United Kingdom. RCOG Press.

Marks MN, Wieck A, Checkley SA, et al (1991) Life stress and postpartum psychosis: a preliminary report. British Journal of Psychiatry, 158 (suppl 10): $45-9$.

McNeil TF (1988) A prospective study of postpartum psychoses in a high risk group. 4. Relationship to life situation and experience of pregnancy. Acta Psychiatrica Scandinavica; 77: 645-53

Munk-OIsen T, Laursen TM, Pedersen CB, et al (2006) New parents and mental disorders. A population-based register study. JAMA; 296 2582-9

National Collaborating Centre for Mental Health (2006) Bipolar disorder: The Management of Bipolar Disorder in Adults, Children and Adolescents, in Primary and Secondary Care. NICE Clinical Guideline CG38. NICE.

National Collaborating Centre for Mental Health (2007) Antenatal and Postnatal Mental Health. The NICE Guideline on Clinical Management and Service Guidance. NICE Clinical Guideline CG45. British Psychological Society \& Royal College of Psychiatrists.
National Collaborating Centre for Women's and Children's Health (2008) Antenatal Care: Routine Care for the Healthy Pregnant Woman. NICE Clinical Guideline 62. NICE.

Oates M (2003a) Perinatal psychiatric disorders. A leading cause of maternal morbidity and mortality. British Medical Bulletin; 67: 219-29.

Oates M (2003b) Suicide. The leading cause of maternal death. British Journal of Psychiatry; 183: 279-81.

Reed P, Sermin N, Appleby L, et al (1999) A comparison of clinical response to electroconvulsive therapy in puerperal and non-puerperal psychosis. Journal of Affective Disorders; 54: 255-60.

Robertson E, Jones I, Haque S, et al (2005) Risk of puerperal and non-puerperal recurrence of illness following bipolar affective puerperal (post-partum) psychosis. British Journal of Psychiatry, 186: 258-9.

Robertson Blackmore E, Jones I, Doshi M, et al (2006) Obstetric variables associated with bipolar affective puerperal psychosis. British Journal of Psychiatry, 188: 32-6.

Sharma V, Smith A, Khan M (2004) The relationship between duration of labour, time of delivery, and puerperal psychosis. Journal of Affective Disorders; 83: 215-20.

Stewart DE, Klompenhouwer JL, Kendell RE, et al (1991) Prophylactic lithium in puerperal psychosis. The experience of three centres. British Journal of Psychiatry, 58: 393-7.

Terp IM, Mortensen PB (1998) Post-partum psychoses. Clinical diagnoses and relative risk of admission after parturition. British Journal of Psychiatry, 172: 521-6.

Viguera AC, Nonacs R, Cohen LS, et al (2000) Risk of recurrence of bipolar disorder in pregnant and nonpregnant women after discontinuing lithium maintenance. American Journal of Psychiatry, 157: 179-84.

Viguera AC, Whitfield T, Baldessarini RJ, et al (2007a) Risk of recurrence in women with bipolar disorder during pregnancy. Prospective study of mood stabilizer discontinuation. American Journal of Psychiatry, 164: 1817-24.

Viguera AC, Newport DJ, Ritchie J, et al (2007b) Lithium in breast milk and nursing infants. Clinical implications. American Journal of Psychiatry, 164: $342-5$.

World Health Organization (1974) Mental Disorders: Glossary and Guide to their Classification in Accordance with the Eighth Revision of the International Classification of Diseases (ICD-8). WHO.

World Health Organization (1978) Manual of the International Statistical Classification of Diseases, Injuries and Causes of Death (9th Revision) (ICD-9). WHO

World Health Organization (1992) The ICD-10 Classification of Diseases Clinical Descriptions and Diagnostic Guidelines. WHO.

\section{MCOs \\ a 1 week \\ b 1 month \\ c 6 months \\ d 1 year \\ e 3 months.}

1 The postpartum onset specifier in DSM-IV identifies postpartum episodes as those with an onset following delivery within:

2 The proportion of deliveries from which puerperal psychosis follows is:

a 1 in 10

b 1 in 100

c 1 in 200

d 1 in 1000

e 1 in 10000 .
3 Definition of puerperal psychosis would usually include:

a an acute confusional state in the context of alcohol withdrawal

b the continuation of a chronic schizophrenic illness into the postpartum period

c the onset of a severe obsessive-compulsive illness in the first 2 weeks following delivery

$d$ an episode of major depression with onset in the first postpartum month

e acute onset in the first postpartum week of an episode with marked manic and psychotic features.

4 The risk of recurrence of bipolar affective puerperal psychosis after future deliveries is about:
a $5 \%$
b $25 \%$
c $50 \%$
d $90 \%$
e $100 \%$.

5 In the treatment of puerperal psychosis:

a the woman should never breastfeed

b admission to hospital is rarely required

c electroconvulsive therapy is contraindicated

d a woman who has experienced an episode should be advised about both the risk of postpartum and non-postpartum recurrence

$\mathrm{e}$ it is not possible to identify women at high risk of puerperal psychosis antenatally. 